\title{
Gravitational baryogenesis in running vacuum models
}

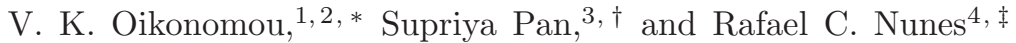 \\ 1 Tomsk State Pedagogical University, 634061 Tomsk, Russia \\ ${ }^{2}$ Laboratory for Theoretical Cosmology, Tomsk State University of \\ Control Systems and Radioelectronics (TUSUR), 634050 Tomsk, Russia \\ ${ }^{3}$ Department of Physical Sciences, Indian Institute of Science Education \\ and Research-Kolkata, Mohanpur-741246, West Bengal, India \\ ${ }^{4}$ Departamento de Fúsica, Universidade Federal de Juiz de Fora, 36036-330, Juiz de Fora, MG, Brazil
}

\begin{abstract}
We study the gravitational baryogenesis mechanism for generating baryon asymmetry in the context of running vacuum models. Regardless if these models can produce a viable cosmological evolution, we demonstrate that they produce a non-zero baryon-to-entropy ratio even if the universe is filled with conformal matter. This is a sound difference between the running vacuum gravitational baryogenesis and the Einstein-Hilbert one, since in the latter case, the predicted baryon-to-entropy ratio is zero. We consider two well known and most used running vacuum models and show that the resulting baryon-to-entropy ratio is compatible with the observational data. Moreover, we also show that the mechanism of gravitational baryogenesis may constrain the running vacuum models.
\end{abstract}

PACS numbers: 98.80.-k, 95.36.+x, 95.35.+d

\section{INTRODUCTION}

The dynamics of the universe is yet a mysterious and unpredictable chapter in modern physics. The addition of late time accelerated expansion of the universe as a new chapter to its dynamical history has been the central theme for the last couple of years. To account such late accelerated expansion, usually one adds a new degrees of freedom associated with an exotic component called dark energy [1] in the framework of general relativity ${ }^{1}$. The simplest dark energy candidate that accommodates the astronomical data quite well is the $\Lambda$-cosmology, where $\Lambda>0$, is the time independent constant. However, the $\Lambda$ term which is equivalent to the vacuum energy density, $\rho_{\Lambda}=\Lambda / 8 \pi G(G$ is the Newton's gravitational constant), leads to the cosmological constant problem that has remain been a serious issue in modern cosmology [12]. Motivated by the cosmological constant problem, a series of alternative cosmological theories have been proposed in the literature, see [1], that gained a massive interest in the scientific community so far. Amongst a class of distinct cosmologies, in this work we are interested to discuss the models where the vacuum energy is dynamical, i.e. when $\rho_{\Lambda}=\rho_{\Lambda}(t)$. In other words, when the cosmological constant $\Lambda$, is replaced by a time dependent term $\Lambda(t)$. From the phenomenological ground this kind of models have been accepted as consistent cosmological theories and in the last couple of years are very well explored in detail [13 31]. In such models the equation of state of the vacuum is strictly equal to ' -1 ', but the energy density of vacuum is variable throughout the history of the universe. However, here we shall focus on a more particular class of dynamical vacuum models, known as the running vacuum models (RVMs).

The RVM models in agreement with the observational data (32] and references there) have the capability to describe the dynamical histroy of the universe in an effective way. In fact, the RVM models are quite good in compared to some other phenomenological models as discussed in Refs. [33 37]. Further, it is also interesting to note that this class of models carry an explanation to the time evolution of the fundamental constants of nature, see [38, 39] and also Ref. [40, 41] for a review on RVMs. We remark that very recently it has been argued that current observational data might favor the dynamical nature of the vacuum [42 [48]. The motivation of the current work is not to investigate whether the RVMs are the alternative description to some other existing cosmological theories, but to investigate the feasibility of the gravitational baryogenesis mechanism in such models.

\footnotetext{
*Electronic address: v.k.oikonomou1979@gmail.com

$\dagger$ Electronic address: span@iiserkol.ac.in

‡Electronic address: rafadcnunes@gmail.com

${ }^{1}$ However, it deserves to be mentioned that the theory of gravitational modifications has also been found to explain the late time accelerated expansion without any need of dark energy [2]11].
} 
It is well known that baryon asymmetry is one of the most persisting problems in modern theoretical physics and cosmology, and it refers to the observed excess of matter over anti-matter. The existing observational data, mainly coming from the cosmic microwave background [49] in conjunction with the successful predictions of Big Bang nucleosynthesis, indicate that matter overwhelms anti-matter. Towards this research line, the gravitational baryogenesis mechanism was proposed some time ago in Ref. [50] in order to explain the excess of matter over anti-matter in the observable universe. Ever since, many modifications or generalizations of the original gravitational baryogenesis mechanism, have been proposed in the literature, see for example [50 62]. In this paper we adopt the approach of the original gravitational baryogenesis mechanism proposed in [50], and we investigate what is the predicted baryon-to-entropy ratio in the context of running vacuum theories. Thus, we considered two well known and most used running vacuum models, the essential features of which we presented in some detail in the next section. For these models we have calculated the baryon-to-entropy ratio and investigated under which conditions the predicted baryon-to-entropy ratio can be compatible with the observed value. The most interesting and the important finding in this work is that the baryon-to-entropy ratio produced by the gravitational baryogenesis mechanism in the presence of a variable vacuum energy is non-zero even in the case that relativistic massless matter is the only matter ingredient of the universe, with regard to perfect fluids. This is different from the Einstein-Hilbert gravity case, where in the presence of radiation matter fluids, the predicted baryon-to-entropy ratio coming from gravitational baryogenesis mechanism is zero, as was showed in [50]. In addition to this result, we also discuss that the compatibility of the models with observations can be achieved. In fact, we find that the observed baryon-to-entropy ratio may be used to constrain the class of RVMs.

This paper is organized as follows: In Section Пw we present the theoretical framework of running vacuum theory and describe the models we shall use. Also, we fix the geometric conventions and explain some feature of running vacuum theories, essential to the later sections. In Section III we briefly discuss the gravitational baryogenesis mechanism where we assume that it generates the baryon asymmetry and calculate the predicted baryon-to-entropy ratio for the running vacuum models of the previous section. In addition, we present some general considerations regarding the predicted baryon-to-entropy ratio in the case that the only perfect fluid present is radiation. Moreover, we compare the results of the running vacuum theory with the Einstein-Hilbert case and investigate in which cases the results are compatible with observations. Finally, the conclusions containing our findings follow at the end of the paper.

\section{DYNAMICAL VACUUM MODELS}

In the present work we are interested in investigating the gravitational baryogenesis mechanism in the context of running vacuum models where the background geometry is described by a spatially flat Friedmann-LemaittreRobertson-Walker (FLRW) line element $d s^{2}=-d t^{2}+a(t)^{2} \sum_{i=1}^{3}\left(d x^{i}\right)^{2}, a(t)$ being the expansion scale factor of the universe. Recently, a number of cosmological models inspired from a variable vacuum energy density have argued that this class of models have better fit with the observational data in comparison to the case of a rigid value to the cosmological constant [2 48]. In the literature there exists various proposals of dynamical vacuum models, but in the present work we follow the approach of [26 31], where the scaling evolution of the vacuum energy occurs through a renormalization group equation for the cosmological constant.

In a spatially flat FLRW universe, the Einstein's equations for a perfect fluid plus a dynamic vacuum energy are given by

$$
\begin{array}{r}
H^{2}=\frac{8 \pi G}{3}\left[\rho+\rho_{\Lambda}\right], \\
2 \dot{H}+3 H^{2}=-8 \pi G\left[p+p_{\Lambda}\right],
\end{array}
$$

where the "dot" represents the differentiation with respect to the cosmic time; $H=\dot{a} / a$ is the Hubble rate; $p, \rho$ are respectively the pressure and the energy density of the perfect fluid with equation of state $p=w \rho, w$ being the equation of state parameter of the perfect fluid, and $\rho_{\Lambda}=\Lambda(H) / 8 \pi G$ is the vacuum energy density associated with the time dependent $\Lambda(H)$, and $p_{\Lambda}\left(=-\rho_{\Lambda}\right)$ is the pressure of the vacuum energy density.

The continuity equation for the total energy density $\rho_{t o t}=\rho+\rho_{\Lambda}$ is written as,

$$
\dot{\rho}+3 H(1+w) \rho=-\dot{\rho}_{\Lambda},
$$

which can also be obtained by using (10) and (2). This conservation equation can also be cast as follows,

$$
\dot{H}+\frac{(1+w)}{2}\left[3 H^{2}-\Lambda(H)\right]=0,
$$


which is the master equation to understand the dynamics of the universe in the context of RVMs. Certainly, to go ahead one needs an expression for $\Lambda(H)$. A series of running vacuum models confronted with observational data can be found in [63]. Thus, being motivated from the previous studies, we recall the most used RVMs in the literature as follows.

The first RVM that we introduce is given by [34, 35, 40, 41]

$$
\operatorname{RVMI}: \Lambda(H)=\alpha+3 \beta H^{2}+3 \delta \frac{H^{n+2}}{H_{I}^{n}},
$$

where $n$ is any positive integer; $H_{I}$ is the Hubble parameter at the inflation scale, $\alpha, \beta, \delta$ are free and dimensionless parameters of the model. The parameter $\alpha$ dominates at low energy (late times), and without any loss of generality it can be fixed/interpreted as the current value of the vacuum energy density ${ }^{2}$. The parameter $\delta$ can be absorbed by the undetermined scale $H_{I}$. Although the inflation scale is undetermined, but however, it is well motivated so that one can consider this to occur on the scales of the order of $10^{16}-10^{19} \mathrm{GeV}$, for example. Thus, in general, the third term in the right hand side of eqn. (5) is predominant during inflation and despicable on the other cosmological eras (radiation, matter and late times), since $H_{I}$ is substantially greater than $H$ in other eras. The parameter $\beta$ can be determined from observations and this parameter quantifies the dynamic character of the vacuum energy. The model above is consistent with the general covariance and can exit from the early inflationary era to the isotropic and homogeneous radiation dominated phase [35]. This class of models can also give an estimation of the ratio of early and present vacuum energy density [34]. We note that the model (5) cannot be analytically solved for arbitrary $n$, but however, the gravitational baryogenesis mechanism can constrain this $\beta$-parameter under certain conditions, that we will show later.

Another model we shall discuss is $\underline{34}, 35,40,41]$

$$
\operatorname{RVMII}: \Lambda(H)=\Lambda_{0}+3 \nu\left(H^{2}-H_{0}^{2}\right),
$$

where $\Lambda_{0}$ is the current value of the vacuum energy density and $\nu$ is a dimensionless parameter. The parameter $\nu$ has been constrained using the observational data which turns out to be of the order of $\nu \sim \mathcal{O}\left(10^{-3}\right)$ 42 44. From a recent study [64], the authors have shown that the growth index can be filed to $\gamma_{\Lambda(H)}=\frac{6+3 \nu}{11-12 \nu}$, extending the growth index of the matter perturbations to RVMs. For a recent analysis of this model including both background and perturbations analysis (including also full CMB Planck likelihood), we also refer to [65], where $\nu \sim \mathcal{O}\left(10^{-4}\right)$ is reported.

In the following sections we shall discuss the implications of these models on the gravitational baryogenesis scenario and we shall investigate when the results are compatible with the observations.

\section{GRAVITATIONAL BARYOGENESIS IN RUNNING VACUUM MODELS}

The gravitational baryogenesis mechanism used in [50], makes use of one of the well known Sakharov criteria for baryogenesis, and it is based on the existence of a $\mathcal{C P}$-violating interaction, which has the following form [50],

$$
\frac{1}{M_{*}^{2}} \int \mathrm{d}^{4} x \sqrt{-g}\left(\partial_{\mu} R\right) J^{\mu}
$$

As was stressed in [50], terms of the form (77) may occur from higher order interaction originating in the complete underlying effective theory which controls the high energy regime. The current term is $J^{\mu}$ and it stands for the baryon matter current, the parameter $M_{*}$ represents the cutoff scale of the effective theory while $g$ and $R$ are respectively the trace of the metric tensor $g_{\mu \nu}$ and the Ricci scalar. As was demonstrated in [50], the resulting baryon-to-entropy ratio for the $\mathcal{C P}$ violating interaction of Eq. (7) is equal to,

$$
\frac{n_{B}}{s} \simeq-\left.\frac{15 g_{b}}{4 \pi^{2} g_{*}} \frac{\dot{R}}{M_{*}^{2} T}\right|_{T_{D}}
$$

\footnotetext{
${ }^{2}$ When assessed at present time, the constant $\alpha$ relates to $\alpha=\Lambda_{0}-3 \beta H_{0}^{2}$ (the third term in the right hand side of (5) can be safely neglected since the scale of $H_{I}$ is much much larger than $H$ at present). Since $\alpha$ is an arbitrary constant, hence, without any loss of generality, we can do $\alpha=\bar{\Lambda}_{0}=\Lambda_{0}-3 \beta H_{0}^{2}$. Therefore, the contribution from $\alpha$ is despicable during very early times.
} 
where $T_{D}$ denotes the decoupling temperature, $g_{b}$ is the number of the intrinsic degrees of freedom of the baryons, and $g_{*}$ is the total number of the degrees of freedom corresponding to effectively massless particles.

An important assumption that needs to be taken into account is that the vacuum decays "adiabatically", that means the specific entropy of the massless particles (entropy per massless particle) remains constant irrespective of the total entropy, which may increase. In that case, some equilibrium relations are hold not all [66], for instance the energy density versus temperature $\left(\rho_{r} \propto T_{r}^{4}\right)$, particle number versus temperature $\left(n_{r} \propto T_{r}^{3}\right)$, but $T_{r}$ does not follow the usual relation $T_{r} \propto a(t)^{-1}$. Following the above line of research the temperature of the massless particle or radiation follows [67]

$$
\rho_{r}=\frac{\pi^{2}}{30} g_{*} T_{r}^{4}
$$

where $g_{*}$ is defined previously.

Let us find an analytic expression for the baryon-to-entropy ratio by using the running vacuum equations of motion. The Ricci scalar $R$, in this spacetime can be calculated to be

$$
R=-8 \pi G(1-3 w) \rho-32 \pi G \rho_{\Lambda}
$$

where we assumed the presence of a perfect fluid with equation of state $p=w \rho$, in which $w$ is the equation of state parameter and it is constant. The expression in eq. (10) is very relevant for the calculation of the baryon-toentropy ratio (8), since the first derivative of the Ricci scalar with respect to the cosmic time appears in eq. (8). By differentiating eq. (10) with respect to the cosmic time we obtain,

$$
\dot{R}=-8 \pi G(1-3 w) \dot{\rho}-32 \pi G \dot{\rho}_{\Lambda}
$$

and by using the continuity equation (3), one may express the first derivative of the perfect fluid energy density $\dot{\rho}$ as a function of $\rho$ and $\dot{\rho}_{\Lambda}$. However, in the following sections we shall focus on the case where the perfect fluid is purely radiation, so in this case $w=1 / 3$.

\section{Baryogenesis in the Conformal Case}

There is a sound difference between the Einstein-Hilbert gravitational baryogenesis and the running vacuum gravitational baryogenesis, for the particular case that the perfect fluid content of the universe is a radiation fluid. In the Einstein-Hilbert case, the Einstein equations have the following form,

$$
R=-8 \pi G(1-3 w) \rho
$$

so the first derivative of the Ricci scalar is, $\dot{R}=-8 \pi G(1-3 w) \dot{\rho}$, and effectively for the a radiation fluid, the baryonto-entropy ratio (8) is zero. In the running vacuum case, the first derivative of the Ricci scalar is given in Eq. (11), and for $w=1 / 3$, this becomes,

$$
\dot{R}=-32 \pi G \dot{\rho}_{\Lambda}
$$

which can be further written as,

$$
\dot{R}=-4 \dot{\Lambda}(H)
$$

where we used $\rho_{\Lambda}=\Lambda(H) / 8 \pi G$. Therefore, even in the conformal case, the resulting baryon-to-entropy ratio is non-zero in the running vacuum case and it is equal to,

$$
\left.\frac{n_{B}}{s} \simeq \frac{15 g_{b}}{\pi^{2} g_{*}} \frac{\dot{\Lambda}(H)}{M_{*}^{2} T}\right|_{T_{D}} .
$$

We remark that if $\Lambda$ is constant, then from eq. (15) one finds that the baryon-to-entropy ratio is zero, which is the usual case. So, effectively, by having the particular form of the running vacuum function $\Lambda(H)$, one can easily find the baryon-to-entropy ratio from Eq. (15). In the following sections we shall concentrate on two particular RVMs (5) and (6) that have been widely investigated in the literature. 


\section{A. Running vacuum model I}

Let us first consider the first RVMs I defined in eq. (5), for which from eq. (4) becomes

$$
\dot{H}+\frac{3(1+w)}{2} H^{2}\left[1-\beta-\frac{\alpha}{3 H^{2}}-\delta\left(\frac{H}{H_{I}}\right)^{n}\right]=0
$$

Evaluating at the early time, the dynamics is governed by

$$
\dot{H} \simeq-\frac{3(1+w)}{2} H^{2}\left[1-\beta-\left(\frac{H}{H_{I}}\right)^{n}\right],
$$

which shows that for $H \simeq H_{I}(1-\beta)^{1 / n}$ (where we must have $(1-\beta)>0$ ), the inflationary period can be realized. As mentioned above, the factor $\delta$ was absorbed in $H_{I}$, so that it can be fixed to unity without any loss of generality (if the scale of inflation is not precisely known). This possibility has already been found in [36], but our motivation is to investigate the baryogenesis mechanism in this context. If however, the de Sitter solution is considered, the predicted baryon-to-entropy ratio in eq. (15) is zero, since in this case the vacuum energy is constant. Therefore, may be one can think of some time dependent solution for $\Lambda(H)$ that could account for the early inflationary era and finally at late time it can survive as a solution to the observed accelerated expansion. When evaluated at early times, the second and third term in the right hand side in eq. (16) can be omitted ${ }^{3}$

$$
\dot{H} \simeq-\frac{3(1+w)(1-\beta)}{2} H^{2}
$$

which can be analytically solved and the result is,

$$
H(t) \simeq \frac{2}{3(1+w)(1-\beta) t},
$$

where without any loss of generality and for simplicity we assume the integration constant to be zero. Within this scenario, the function $\Lambda(H)$ is approximately equal to $\Lambda(H) \simeq 3 \beta H^{2}$, that is, a pure dynamic vacuum. Thus, the resulting baryon-to-entropy ratio in the conformal case, where $w=1 / 3$, can be easily found by using eq. (15). We find,

$$
\left.\frac{n_{B}}{s} \simeq \frac{15 g_{b}}{4 \pi^{2} g_{*}} \frac{6 \beta H \dot{H}}{M_{*}^{2} T}\right|_{T_{D}} .
$$

By using Eqs. (11) and (9), we can express the decoupling time $t_{D}$ as a function of the decoupling temperature $T_{D}$, resulting to

$$
t_{D}=\sqrt{\frac{90 g_{b}}{2 \pi G}}\left(\frac{1}{3(1+w)(1-\beta) \pi T_{D}}\right)
$$

By combining Eqs. (19) and (20), the resulting expression for the baryon-to-entropy ratio is given by

$$
\frac{n_{B}}{s} \simeq \frac{120 g_{b}}{g_{*} M_{*}^{2}}\left(\frac{2 \pi G}{90 g_{b}}\right)^{3 / 2} 3(1+w)(1-\beta) \pi^{2} T_{D}^{2}
$$

\footnotetext{
3 The constant $\alpha$ is despicable in early times since this factor plays like $\alpha=\Lambda_{0}$, the current value of vaccum energy density which is very small. Moreover, the contribution of the term $H^{n} / H_{I}$, can also be discarded during the radiation era.
} 
Thus, from eq. (22) one can conclude that for RVM I, the calculated baryon-to-entropy ratio should be nonzero since $\beta \neq 1$. Now, one can find an approximate value of $n_{B} / s$, using the values of the free parameters in a reasonable way. To illustrate this notion, we consider a particular example that will reflect the nonzero baryon-to-entropy ratio. We consider the cutoff scale to be $M_{*}=10^{12} \mathrm{GeV}$, in addition that the decoupling temperature $\mathcal{T}_{D}$ is equal to $\mathcal{T}_{D}=M_{I}=2 \times 10^{16} \mathrm{GeV}$, with $M_{I}$ being the upper bound for the fluctuations constraints on tensor-modes of the inflationary scale, and also that $g_{b} \simeq \mathcal{O}(1), g_{*} \simeq 106$, which is the total number of effective massless particles at early times that is well known.

At this point it is worth discussing the values assigned to the parameters $M_{*}, M_{I}$ and $T_{D}$. Recall that $M_{*}$ is the scale that the operator appearing in Eq. (7) becomes effective, $M_{I}$ is the inflationary scale, and $T_{D}$ is the decoupling temperature. The cutoff scale $M_{*}$ in a gravitational context is expected to be much lower than the Planck scale, so we assumed for example $M_{*}=10^{12} \mathrm{GeV}$, but in principle it can take alternative values. Now for the purposes of this paper we shall assume that $M_{I} \sim \mathcal{T}_{D}$, which is also the choice made in Ref. [50]. This choice significantly constrains the decoupling temperature $\mathcal{T}_{D}$, since the inflationary scale is constrained as follows $M_{I} \leq 3.3 \times 10^{16} \mathrm{GeV}[50]$. Hence, the choice $\mathcal{T}_{D}=M_{I}=2 \times 10^{16} \mathrm{GeV}$, is compatible with the above constraint.

Now, with the choices made above, the resulting baryon-to-entropy ratio becomes very sensible to the parameter $\beta$. For different values of $\beta$, we can calculate the baryon-to-entropy ratio. However, for a typical value of $\beta=0.999$, the resulting baryon-to-entropy ratio is $n_{B} / s \simeq 17.9316 \times 10^{-11}$, which is compatible with the observed value $n_{B} / s \simeq$ $9 \times 10^{-11}$. On the other hand, for $\beta \gtrsim 1$, the resulting baryon-to-entropy ratio becomes "negative", which means that, the model with $\beta \gtrsim 1$ predicts an excess of anti-matter over matter, which is unphysical. Thus, it is evident that the mechanism of gravitational baryogenesis constrains the RVMs under consideration. Note that we are considering the conformal case, i.e., $w=1 / 3$. However, one may note that it is possible to obtain the nonzero $n_{B} / s$ for some other specific choices of the free parameters, where we can take different values of $\beta<1$. We can note that for $\beta=0.999$, we have $\Lambda_{0} \simeq 3 H_{0}^{2}$ at late times within this context addressed here. This value has the same order of magnitude with the standard case $\Lambda_{0} \simeq 2 H_{0}^{2}$ (assuming $\Omega_{\Lambda}=0.70$ and natural units). Therefore, the class RVM I can produce a non-zero and physically acceptable baryon-to-entropy ratio within this context.

\section{B. Running vacuum model II}

Let us now calculate the predicted baryon-to-entropy ratio for RVM II given by eq. (6), for the conformal matter fluid case. Here, the Hubble rate can be found analytically and it reads,

$$
H=\sqrt{\frac{\Omega_{\Lambda 0}-\nu}{1-\nu}} \tanh \left[\frac{3(1+w)}{2} H_{p} \sqrt{(1-\nu)\left(\Omega_{\Lambda 0}-\nu\right)}\left(t-t_{p}\right)+B\right]
$$

where $H_{p}=H\left(t=t_{p}\right), B=\tanh ^{-1}\left(\sqrt{\frac{1-\nu}{\Omega_{\Lambda 0}-\nu}} H_{p}\right)$. Further, one can integrate the above equation to find the scale factor as,

$$
a=a_{p}\left[\frac{\cosh \left(\frac{3}{2}(1+w) H_{p} \sqrt{(1-\nu)\left(\Omega_{\Lambda 0}-\nu\right)}\left(t-t_{p}\right)+B\right)}{\cosh B}\right]^{\frac{2}{3(1+w) H_{p}(1-\nu)}} .
$$

We can find the leading order expression for the decoupling time as a function of the decoupling temperature, which in this case is,

$$
t_{D}=\frac{45 \mathcal{C}_{1}-4 \pi G g_{*} T_{D}^{4}}{\mathcal{C}_{2}}
$$

where the parameters $\mathcal{C}_{1}$ and $\mathcal{C}_{2}$ are defined as

$$
\mathcal{C}_{1}=\frac{\left(\nu-\Omega_{\Lambda_{0}}\right) \tanh \left[B-\frac{3}{2} H_{p} t_{p}(1+w) \sqrt{(\nu-1)\left(\nu-\Omega_{\Lambda_{0}}\right)}\right]^{2}}{\nu-1},
$$




$$
\begin{gathered}
\mathcal{C}_{2}=\frac{3\left(\left(\nu-\Omega_{\Lambda_{0}}\right)\left(-H_{p}(1+w) \sqrt{(\nu-1)\left(\nu-\Omega_{\Lambda_{0}}\right)} \tanh \left[B-\frac{3}{2} H_{p} t_{p}(1+w) \sqrt{(\nu-1)\left(\nu-\Omega_{\Lambda_{0}}\right)}\right]\right)\right)}{\nu-1} \\
+\frac{\left.3\left(H_{p}(1+w) \sqrt{(\nu-1)\left(\nu-\Omega_{\Lambda_{0}}\right)} \tanh \left[B-\frac{3}{2} H_{p} t_{p}(1+w) \sqrt{(\nu-1)\left(\nu-\Omega_{\Lambda_{0}}\right)}\right]^{3}\right)\right)}{\nu-1} .
\end{gathered}
$$

Now, for this model it can be shown that the function $\dot{R}$ is equal to,

$$
\dot{R} \simeq-24 \nu\left[\mathcal{A}_{1}-\frac{\mathcal{A}_{2}}{\mathcal{C}_{2}}\left(\mathcal{C}_{1}-\frac{4 \pi G g_{*} T_{D}^{4}}{45}\right)\right],
$$

where the parameters $\mathcal{A}_{1}$ and $\mathcal{A}_{2}$ can be calculated as

$$
\begin{aligned}
\mathcal{A}_{1}= & \frac{3 H_{p}(1+w)\left(\nu-\Omega_{\Lambda_{0}}\right) \sqrt{(\nu-1)\left(\nu-\Omega_{\Lambda_{0}}\right)} \operatorname{sech}\left[B-\frac{3}{2} H_{p} t_{p}(1+w) \sqrt{(\nu-1)\left(\nu-\Omega_{\Lambda_{0}}\right)}\right]^{2}}{2(\nu-1)} \\
& \times \tanh \left[B-\frac{3}{2} H_{p} t_{p}(1+w) \sqrt{(\nu-1)\left(\nu-\Omega_{\Lambda_{0}}\right)}\right] \\
\mathcal{A}_{2}= & \frac{9}{4}\left(H _ { p } ^ { 2 } ( 1 + w ) ^ { 2 } ( \nu - \Omega _ { \Lambda _ { 0 } } ) ^ { 2 } \left(-\operatorname{sech}\left[B-\frac{3}{2} H_{p} t_{p}(1+w) \sqrt{(\nu-1)\left(\nu-\Omega_{\Lambda_{0}}\right)}\right]^{2}\right.\right. \\
+ & \left.3 \operatorname{sech}\left[B-\frac{3}{2} H_{p} t_{p}(1+w) \sqrt{(\nu-1)\left(\nu-\Omega_{\Lambda_{0}}\right)}\right]^{2} \tanh \left[B-\frac{3}{2} H_{p} t_{p}(1+w) \sqrt{(\nu-1)\left(\nu-\Omega_{\Lambda_{0}}\right)}\right]^{2}\right)
\end{aligned} .
$$

Therefore, the resulting baryon-to-entropy ratio for RVM II is given by

$$
\frac{n_{B}}{s} \simeq \frac{90 \nu g_{g}}{\pi g_{*} M_{*}^{2} T_{D}}\left[\mathcal{A}_{1}-\frac{\mathcal{A}_{2}}{\mathcal{C}_{2}}\left(\mathcal{C}_{1}-\frac{4 \pi G g_{*} T_{D}^{4}}{45}\right)\right] .
$$

In this case we assume the cutoff scale $M_{*}=10^{12} \mathrm{GeV}$, in addition to that, the decoupling temperature $T_{D}$ to $\mathcal{T}_{D}=M_{I}=0.23 \times 10^{13} \mathrm{GeV}, g_{b} \simeq \mathcal{O}(1), t_{p} \simeq 10^{-20} \mathrm{sec}, g_{*} \simeq 106, H_{p} / \Omega_{\Lambda_{0}}=10^{-6}$, and $\nu=0.001{ }^{4}$.

Notice that, as in the previous model, we assume that $M_{*}=10^{12} \mathrm{GeV}$, however, in this case we made the choice $\mathcal{T}_{D}=M_{I}=0.23 \times 10^{13} \mathrm{GeV}$, which is compatible with the observational constraint on the inflationary scale $M_{I} \leq$ $3.3 \times 10^{16} \mathrm{GeV}[50]$.

By using the above mentioned values for the parameters, we find that the baryon-to-entropy ratio is approximately equal to $n_{B} / s \simeq 7.29947 \times 10^{-11}$, which is again compatible with the observed value of $n_{B} / s \simeq 9 \times 10^{-11}$.

Therefore, for the RVM II model too, even in the conformal case, the predicted baryon-to-entropy ratio is non-zero. In principle, the function $\Lambda(H)$ crucially affects the predicted baryon-to-entropy ratio. Thus, it is possible that the gravitational baryogenesis mechanism may pose some constraints on the general class of models where the vacuum energy is variable.

\section{CONCLUSIONS}

In this paper we investigated the gravitational baryogenesis mechanism in the context of running vacuum models, regardless if these scenarios can produce a viable baryon-to-entropy ratio. We found that there is a sound difference between the Einstein-Hilbert baryon-to-entropy ratio and the one corresponding the scenarios with running vacuum energy. Particularly, when the universe is filled with conformal matter fluids, the baryon-to-entropy ratio is zero in the Einstein-Hilbert case, whereas in the running vacuum scenario the predicted baryon-to-entropy ratio is non-zero. We calculated in some detail the baryon-to-entropy ratio for two well known RVM models and we demonstrated that the resulting picture is compatible with the observational data. From both the models, we came up with the conclusion

\footnotetext{
4 This value is fixed from the results in 42,44$].$
} 
that in the case of RVMs the resulting baryon-to-entropy ratio is non-zero even in the presence of conformal matter. We also observed that the gravitational baryogenesis mechanism can be used to constrain the RVM models. In general, a nonzero ratio of baryon-to-entropy is sensitive to the values of $\beta$ (RVM I) and $\nu$ (RVM II). Thus, it is clear that cosmological models with running vacuum energy carry a possible explanation to the non-zero baryon-to-entropy ratio in our universe and conversely the observed value of the baryon-to-entropy ratio can also be used to constrain the RVM models as well. Although the current work focuses on two well known models of running vacuum energy, but it would be interesting to study the cosmological scenarios driven by another models in order to measure the baryogenesis effect which could potentially impose some constraints on these phenomenological scenario.

Finally, we close the work drawing a possible connection of this scenario with the extended gravity theories. It is well known that the physics of the universe at early times constitutes a great laboratory to explore physics beyond the standard cosmological model. For instance, torsion in $f(T)$ theories of gravity can be constrained by big bang nucleosynthesis [68], modifications on the evolution of the thermal relic particles (particles like WIMPs (weakly interactive massive particles)) within $f(R)$ gravity context are also taken into account in [69]. Thus, it is worth noting that the nonzero ratio of baryon-to-entropy of the universe could be a potential quantity to constrain extended theories of gravity too.

\section{Acknowledgments}

The authors gratefully acknowledge the referees for their comments to improve the work. The authors also thank Prof. J. A. S. Lima for some useful comments. This work is supported respectively by the "Min. of Education and Science of Russia" (V.K.O) and "SERB-NPDF (File No: PDF/2015/000640)", Govt. of India (S.P).

[1] K. Bamba, S. Capozziello, S. Nojiri and S. D. Odintsov, Astrophys. Space Sci. 342 (2012) 155 arXiv:1205.3421 [gr-qc]].

[2] S. Nojiri and S. D. Odintsov, Phys. Rept. 505, 59 (2011) arXiv:1011.0544 [gr-qc]].

[3] S. Nojiri and S. D. Odintsov, Int. J. Geom. Meth. Mod. Phys. 11, 1460006 (2014) arXiv:1306.4426 [gr-qc]].

[4] S. Nojiri, S. D. Odintsov and V. K. Oikonomou, arXiv:1705.11098 [gr-qc].

[5] V. Faraoni and S. Capozziello, "Beyond Einstein gravity: A Survey of gravitational theories for cosmology and astrophysics," Fundamental Theories of Physics, Vol. 170, Springer, 2010

[6] S. Capozziello and M. De Laurentis, Phys. Rept. 509, 167 (2011) arXiv:1108.6266 [gr-qc]].

[7] K. Bamba and S. D. Odintsov, Symmetry 7, 220 (2015) arXiv:1503.00442 [hep-th]].

[8] L. Sebastiani and R. Myrzakulov, Int. J. Geom. Meth. Mod. Phys. 12, 1530003 (2015) arXiv:1506.05330 [gr-qc]].

[9] S. D. Odintsov and V. K. Oikonomou, Class. Quant. Grav. 33, 125029 (2016) arXiv:1602.03309 [gr-qc]].

[10] V. K. Oikonomou, Astrophys. Space Sci. 361, 211 (2016) arXiv:1606.02164 [gr-qc]].

[11] Y. F. Cai, S. Capozziello, M. De Laurentis and E. N. Saridakis, Rept. Prog. Phys. 79, 106901 (2016) arXiv:1511.07586 [gr-qc].

[12] S. Weinberg, Rev. Mod. Phys. 61, 1 (1989).

[13] O. Bertolami, Nuovo Cim. B 93, 36 (1986).

[14] M. Reuter and C. Wetterich, Phys. Lett. B 188, 38 (1987).

[15] P. J. E. Peebles and B. Ratra, Astrophys. J. 325, L17 (1988).

[16] J. C. Carvalho, J. A. S. Lima and I. Waga, Phys. Rev. D 46, 2404 (1992).

[17] D. Pavón, Phys. Rev. D 43, 375 (1991).

[18] I. Waga, Astrophys. J. 414, 436 (1993).

[19] J. A. S. Lima and J. M. F. Maia, Phys. Rev. D 49, 5597 (1994).

[20] A. I. Arbab, Class. Quant. Grav. 20 93, (2003) gr-qc/9905066.

[21] A. I. Arbab, JCAP 0305 008, (2003) astro-ph/0212565.

[22] A. I. Arbab, Astrophys. Space Sci. 291 141, (2004) astro-ph/0212145].

[23] A. S. Al-Rawaf and M. O. Taha, Gen. Rel. Grav. 28, 935 (1996).

[24] P. Wang, X.H. Meng, Class. Quant. Grav. 22, 283 (2005) astro-ph/0408495.

[25] M. Szydlowski and A. Stachowski, arXiv:1508.05637 [astro-ph.CO].

[26] I. L. Shapiro and J. Solà, JHEP 0202, 006 (2002) arXiv:hep-th/0012227.

[27] I. L. Shapiro, J. Solà, and H. Stefancic, JCAP 0501, 012 (2005) arXiv:hep-ph/0410095.

[28] I. L. Shapiro and J. Solà, Phys. Lett. B 475, 236 (2000) hep-ph/9910462 .

[29] A. Babic, B. Guberina, R. Horvat and H. Stefancic, Phys. Rev. D 65, 085002 (2002) hep-ph/0111207.

[30] B. Guberina, R. Horvat, H. Stefancic, Phys. Rev. D 67, 083001 (2003) hep-ph/0211184.

[31] I. L. Shapiro, J. Solà, Nucl. Phys. B Proc. Supp. 127, 71 (2004) hep-ph/0305279].

[32] J. Solà, J. C. Perez, and A. G. Valent, arXiv:1703.08218 [astro-ph.CO].

[33] J. Grande, J. Sola, S. Basilakos and M. Plionis, JCAP 1108, 007 (2011) arXiv:1103.4632 [astro-ph.CO]]. 
[34] J. A. S. Lima, S. Basilakos and J. Solà, Mon. Not. Roy. Astron. Soc. 431, 923 (2013) arXiv:1209.2802 [gr-qc]].

[35] E. L. D. Perico, J. A. S. Lima, S. Basilakos and J. Solà, Phys. Rev. D 88, 063531 (2013) arXiv:1306.0591 [astro-ph.CO]].

[36] S. Basilakos, J. A. S. Lima and J. Solà, Int. J. Mod. Phys. D 22, 1342008 (2013) arXiv:1307.6251 [astro-ph.CO]].

[37] S. Basilakos and J. Sola, Mon. Not. Roy. Astron. Soc. 437, 3331 (2014) arXiv:1307.4748 [astro-ph.CO]].

[38] H. Fritzsch and J. Solà, Class. Quant. Grav. 29, 215002 (2012) arXiv:1202.5097 [hep-ph]].

[39] H. Fritzsch, R. C. Nunes, and J. Solà, Eur. Phys. J. C 77, 193 (2017) arXiv:1605.06104 [hep-th]].

[40] J. Solà, J. Phys. Conf. Ser. 453, 012015 (2013) arXiv:1306.1527 [gr-qc]].

[41] J. Solà and A. Gomez-Valent, Int. J. Mod. Phys. D 24, 1541003 (2015) arXiv:1501.03832 [gr-qc]].

[42] J. Solà, A. G. Valent and J. C. Perez, Astrophys. J. 811, L14 (2015) arXiv:1506.05793 [gr-qc]].

[43] J. Solà, A. G. Valent and J. C. Perez, Astrophys.J. 836, 43 (2017) arXiv:1602.02103 [astro-ph.CO]].

[44] J. Solà, A. G. Valent, J. C. Perez and R. C. Nunes, arXiv:1606.00450 [gr-qc].

[45] V. Salvatelli, N. Said, M. Bruni, A. Melchiorri and D. Wands, Phys. Rev. Lett. 113, 181301 (2014) arXiv:1406.7297 [astro-ph.CO]].

[46] C. Pigozzo, S. Carneiro, J.S. Alcaniz, H. A. Borges, and J.C. Fabris, JCAP 1605, 022 (2016) arXiv:1510.01794 [astroph.Co]].

[47] S. Kumar and R. C. Nunes, Phys. Rev. D 94, 123511 (2016) arXiv:1608.02454 [astro-ph.CO]].

[48] S. Kumar and R. C. Nunes, arXiv:1702.02143 [astro-ph.CO].

[49] C. L. Bennett et al. [WMAP Collaboration], Astrophys. J. Suppl. 148, 1 (2003) arXiv:astro-ph/0302207.

[50] H. Davoudiasl, R. Kitano, G. D. Kribs, H. Murayama and P. J. Steinhardt, Phys. Rev. Lett. 93, 201301 (2004) hep-ph/0403019.

[51] G. Lambiase and G. Scarpetta, Phys. Rev. D 74, 087504 (2006) astro-ph/0610367.

[52] G. Lambiase, S. Mohanty and A. R. Prasanna, Int. J. Mod. Phys. D 22, 1330030 (2013) arXiv:1310.8459 [hep-ph]].

[53] G. Lambiase, Phys. Lett. B 642, 9 (2006) hep-ph/0612212.

[54] H. Li, M. z. Li and X. m. Zhang, Phys. Rev. D 70, 047302 (2004) hep-ph/0403281.

[55] L. Pizza, arXiv:1506.08321 [gr-qc].

[56] S. D. Odintsov and V. K. Oikonomou, Phys. Lett. B 760, 259 (2016) arXiv:1607.00545 [gr-qc]].

[57] V. K. Oikonomou and E. N. Saridakis, Phys. Rev. D 94, 124005 (2016) arXiv:1607.08561 [gr-qc]].

[58] E. V. Arbuzova and A. D. Dolgov, JCAP 1706, 001 (2017) arXiv:1702.07477 [gr-qc]].

[59] E. V. Arbuzova and A. D. Dolgov, Phys. Lett. B 769, 171 (2017) arXiv:1612.06206 [gr-qc]].

[60] A. De Simone, T. Kobayashi and S. Liberati, Phys. Rev. Lett. 118, 131101 (2017) arXiv:1612.04824 [hep-ph]].

[61] S. D. Odintsov and V. K. Oikonomou, Europhys. Lett. 116, 49001 (2016) arXiv:1610.02533 [gr-qc]].

[62] V. K. Oikonomou, Grav. Cosmol. 23 , 162 (2017)

[63] A. Gómez-Valent, J. Solà and S. Basilakos, JCAP 1501, 004 (2015) arXiv:1409.7048 [astro-ph.CO]].

[64] S. Basilakos and J. Solà, Phys. Rev. D 92, 123501 (2015) [arXiv:1509.06732 [astro-ph.CO]].

[65] C. Q. Geng, C. C. Lee and L. Yin, arXiv:1704.02136 [astro-ph.CO].

[66] J. A. S. Lima, Phys. Rev. D 54, 2571 (1996) arXiv:gr-qc/9605055.

[67] J. A. S. Lima, S. Basilakos and J. Solà, Eur. Phys. J. C 76, 228 (2016) arXiv:1509.00163 [gr-qc]].

[68] S. Capozziello, G. Lambiase and E. N. Saridakis, arXiv:1702.07952 [astro-ph.CO].

[69] S. Capozziello, V. Galluzzi, G. Lambiase and L. Pizza, Phys. Rev. D 92, 084006 (2015) arXiv:1507.06835 [astro-ph.CO]]. 